\title{
sciendo
}

\section{Incorporating artificial intelligence in knowledge creation processes in organizations}

\author{
Andrei Ștefan NEȘTIAN \\ Alexandru Ioan Cuza University, Iasi, Romania \\ nestian@uaic.ro \\ SilviuMihail TIŢ $\breve{A}$ \\ Alexandru Ioan Cuza University, Iasi, Romania \\ silviutita@yahoo.com \\ Alexandra Luciana GUȚ $\breve{A}$ \\ Alexandru Ioan Cuza University, Iasi, Romania \\ luciana_guta@yahoo.com
}

\begin{abstract}
In organizations, the knowledge creation remained an exclusive attribute of humans until the emergence of artificial intelligence. The role of information systems in organizations expanded continuously, including recording, organizing, processing, storage, dissemination and making available the information, until the revolutionary emergence of artificial intelligence. The role of knowledge creation is no longer exclusive to humans. Artificial intelligence becomes a partner entity of employees in knowledge creation processes in organizations. This article investigates how artificial intelligence can be incorporated in knowledge creation processes. Starting from the study of scientific literature, this article presents concepts and taxonomies associated with the use of artificial intelligence in the processes of knowledge creation in organizations. The study is a first step in a larger research, which aims to identify the main consequences, on employees and organizations, of the incorporation of artificial intelligence in the processes of knowledge creation.
\end{abstract}

Keywords: knowledge creation, artificial intelligence, knowledge management, processes, information systems.

\section{Introduction}

In human history, knowledge creation remained an exclusive attribute of humans until the emergence of artificial intelligence (AI). The history of this change begins with the appearance of computerized information systems, able to process information in different ways than humans do.

Using a computer helps you make complex calculations, helps high speed image processing, helps transmitting a signal via satellites, helps controlling complex production systems or complex warehouse logistics systems, at a level of complexity beyond human ability. In all these cases, the computerized system is processing information and is producing data after the rules set by the programmer and according to the demands of the user. The computer is an extension of the programmer and a tool for its user. Based on this relation between the individual and the computer, the role of information systems in organizations expanded continuously, including recording, organizing, processing, storing, disseminating and making available the information. The limited ability of computers to process information, making them capable of producing a predefined result after using an algorithm for information processing implemented by a programmer, restricted their contribution at knowledge creation in organizations to a role of information suppliers, able to augment in speed and complexity the individual ability to process 
information. Computers are able to produce new data, based on their processing, but these data need to be put into context by individuals in order to produce value in the organization. Computer systems were mostly viewed as computing machines, storages of mass data and used for decision support. Hence, the role of producing knowledge was considered to remain the exclusive privilege of the individuals.

\section{Artificial intelligence}

All these interactions between men and computerized systems have changed with the revolutionary emergence of artificial intelligence. In 1950, the mathematician Alan Turing changed history with a simple, but challenging question: "Can machines think?" Turing's paper "Computing Machinery and Intelligence" (1950), and it's subsequent Turing Test, established the fundamental goal and vision of artificial intelligence (Moor, 2003). Thinking is the main attribute of intelligence, and the use of this verb to describe the operations performed by a machine was a real shock. Introducing the idea of intelligent computing machines was subject to many objections. A renowned objection was formulated by Lady Lovelace, a famous English mathematician and writer, stating that machines cannot originate anything, can never do anything new, can never surprise us (Bringsjord et al., 2001). It was a way of saying that computerized systems, including AI systems, are able to provide us with new data, but they are not able to create new knowledge. Computers and Artificial Intelligence are recognized as partner entities of employees in processing information in organizations, but they are not considered as having the ability to create knowledge. This statement was challenged both in theory and in practice. We will challenge it by exploring the thin philosophical edge between data, information and knowledge, in order to understand whether knowledge creation is or is not a feature of the superior computerized systems called Artificial intelligence.

This new feature, of creating knowledge, is not openly stated in the definitions of AI. Starting from the technical foundations, AI is considered as a program that performs tasks based on algorithms in an intelligent manner (Kusunose et al., 2019). The definition acknowledges the use of algorithms in AI and raises an apparently simple question: what is an intelligent manner of performing a computerized processing? One of the definitions used by the practitioners in this field accepts AI as a computer system able to perform tasks that ordinarily require human intelligence (builtin.com/artificial-intelligence). This statement shows the limits of our understanding of intelligence: we truly recognize as superior intelligence the only one we know: the intelligence of the human species. In EnciclopediaBritanica we find a broader perspective on AI replicating the intelligence of beings: "Artificial intelligence (AI) is the ability of a digital computer or computer-controlled robot to perform tasks commonly associated with intelligent beings" (Copeland, 2019). This broad perspective might allow us to recognize as intelligent even systems less intelligent than humans, but still able to perform simpler tasks, performed by other animals of Earth, such as primates, or by human children. Copeland $(2019, \mathrm{p} 1)$ states that AI is "a term frequently applied to the project of developing systems endowed with the intellectual processes characteristic of humans, such as the ability to reason, discover meaning, generalize, or learn from past experience".

Another definition of AI used by professionals in this field considers that AI makes it possible for machines to learn from experience, adjust to new inputs and perform human-like tasks (www.sas.com/what-is-artificial-intelligence). This perspective is supported by Copeland, 
in EnciclopediaBritanica, in his statement that research in AI has been focused chiefly on the components of human intelligence: learning, reasoning, problem solving, perception, and using language (Copeland, 2019). Akerkar (2019, p.6) follows the same line of arguments stating that AI provides all mechanisms to machines that need to accumulate knowledge and learn from vast amount of data, power of simulating nature-inspired behaviour besides typical intelligent models and algorithm. According to Pfaffenberger (1993, p. 20), this perspective on AI created a scientific and practice field also called "Artificial intelligence", a field dedicated to the computer sciences that intends to perfect the computers by endowing them with some peculiar characteristics of human intelligence, as the capacity to understand natural language and to simulate the reasoning in uncertainty conditions."

\section{Artificial intelligence learning}

Theorists and practitioners in the field consider that AI are machines able to learn. And, since learning is considered to be the acquisition of knowledge or skills through study, experience, or being taught (https://learnersdictionary.com/definition/learning), we can conclude that AI are acquiring knowledge through experience. In fact, one of the main fields of AI applications is called machine learning. AI machines are repeatedly fed data until they learn.

"If we consider learning as any relatively permanent change in behavior that occurs as a result of experience" (Robbins, 2003), we can state that if AI are learning, then we should be able to perceive a change in the way they operate and in the results of their operations which should not be directly generated by the initial form of the algorithms embedded in the design of the AI. Technically this means that we should expect the inner change of the algorithms or other restructuring of the way data are processed leading to a change of the answers AI offers in interaction with reality.

This interaction with reality is highlighted by Spence (1950, p. 161) which characterizes cognitive theories of learning as those that "emphasized the formation and modification of cognitive patterns representative of the relationships in the environment". This implies three levels of intelligent interaction between AI and the environment: sensing reality, representing reality and performing (Akerkar, 2019). Hence, a learning AI will be able to sense reality, to make representations of reality in its processes and to perform actions in a way that reveals inner changes on either of these three layers. In other words, this ability should be seen as AI being able to acquire knowledge from different sources, process information using adaptive rules and later apply that acquired results in different ways in different contexts. Artificial Intelligence is designed to not just seek patterns but also to learn from experience so that they can self-select the appropriate responses according to the situation (Shekhar, 2019).

In order to better understand the AI ability of learning, it is useful to point out what differentiates AI from the regular automation. The similarity between automation and AI is that the creator formulates the algorithms for processing the information. In many respects, the computers are designed to perform automatic information processing. The difference between automation and $\mathrm{AI}$ is that in the case of automation the creator already knows the output of the automation process while in the case of AI the creator doesn't know the output, hence the output can be considered new information, and, after being used in a specific context, it can be considered new knowledge. Shekhar $(2019$, p. 15) states that in automation, "most likely you will be teaching the system all that you already know. In case of automatic, you will be able to easily know the output using sensor readings. But in the case of AI there is always a little bit of 
uncertainty, just like it's there with the human brain". Automatic machines are reproducing a discrete or singular case of human piece of knowledge. The AI does not mimic the result of one particular human cognitive process, the AI tries to mimic the human cognitive process.

Another idea to be addressed in order to understand the learning of AI is the difference between simply memorizing, an ability possessed by all computers, and generalization, the ability to apply past experience to analogous new situations. Having the physical ability to store data is a feature of any computer. But, in order to be able cu create an appropriate answer to a slightly new situation, an AI must process the stored data in an adaptive way, creating an inner result different from all the previously stored solutions. This requires AI to discover meaningful and useful patterns in large volumes of data of any type, including text, images, video and other unstructured data (Akerkar, 2019).

One of the human intellectual abilities is reasoning, based on making inferences. An inference is an idea or conclusion that's drawn from evidence, using the process of reasoning. Copeland (2019, p.1) states that "we learn about some things by experiencing them first-hand, but we gain other knowledge by inference - the process of inferring things based on what is already known. An inference is any process of reasoning from premises to a conclusion" (Copeland, 2019). From a theoretical point of view, inferences are classified as deductive or inductive. In a deductive inference, the truth of the premises guarantees the truth of the conclusion. In an inductive inference, the truth of the premise lends support to the conclusion without giving absolute assurance. Computers are designed to easily produce results based on deductive inferences, which uses logic and mathematics. This is easy since in a deductive inference, we make a prediction of consequences based on a previously demonstrated theory. The theory is the general reference and the observation is the specific element in the inference. For a computer, a general rule of an algorithm stands for the theory, and the input from a user or from a sensor is the specific element, the observation. But, for an AI, the real challenge is to be able to produce inductive inferences. In an inductive inference, we go from the specific to the general. We make many observations, discern a pattern, make a generalization, and infer an explanation or a theory. For this reason, AI are often used for processing big data in order to discover meaningful and useful patterns in large volumes of data of any type, including text, images, video and other unstructured data.

\section{AI abilities of sensing, cognizing and performing}

Akerkar (2019, p. 3) provides a perspective on AI that uses three dimensions of intelligence. They are supposed to be able to sense, to cognize and to perform (Figure 1).

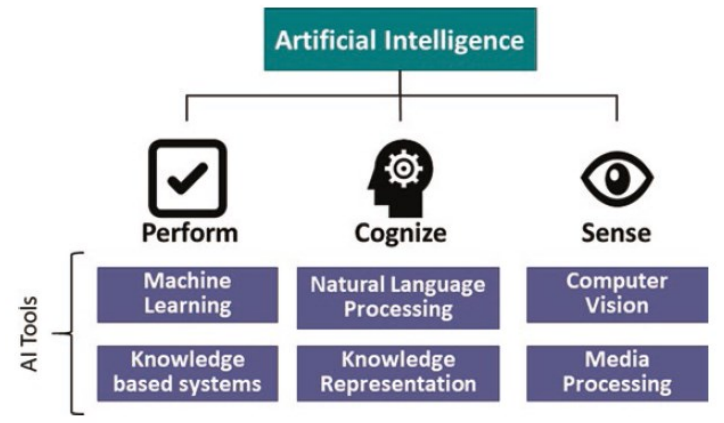

Figure 1. Introduction to Artificial Intelligence

Source: Akerkar, R. (2019). Introduction to artificial intelligence. In Artificial Intelligence for Business 
These three dimensions are based on different tools. For processing images and other sensory inputs, an array of AI applications are focused on media processing. "Whenever intelligence is demonstrated by machines in the surrounding environment, it is referred to as ambient intelligence" (Gams et al., 2019). This sense ability of AI is definitory for what is known as an "intelligent agent": a system that perceives its environment and takes actions to improve its chance of success (Russell \&Norvig, 2016). Lemaignan et al. (2017, p. 45)are stating that many AI techniques are mandated, from visual processing to symbolic reasoning, from task planning to theory of mind building, from reactive control to action recognition and learning.

For achieving abilities to process natural language, an array of AI applications are focused on processing human language, both written or spoken, based on knowledge representation. "Reasonable translation systems are available today for use in restricted context, mainly effective if a little human guidance can be provided to the system. Semantic Web, content and link analysis of web pages, text mining, extraction of specific information from documents, automatic classification and personalized agents hunting for information of interest to a specific individual are some of the active areas today" (Akerkar, 2019). Speech processing has already generated functionally valuable tools. Software tools available now on the market can convert spoken text into machine processable text such as Word document or into vocal commands.

Knowledge-based systems were perhaps the most successful practical branch of AI. Hundreds of tools, commonly labelled expert system shells, were developed in organizations all over the world (Akerkar, 2019). Machine learning applications are also in this category. Machine learning involves the development of computational approaches to automatically make sense of data in dynamic processes made possible through examples and experiences as opposed to predefined rules (Akerkar, 2019). Machine learning uses repetitive sets of experimental data and uses statistical techniques to help the AI learn how to get progressively better at executing a task, without having been specifically programmed for that task. The neural networks contain a number of hidden layers through which the data is processed, allowing the machine to go "deep" in its learning, making connections and weighting input for the best results (https://builtin.com/artificial-intelligence). Machine learning are computer algorithms that learn from sample inputs and apply that learning to make predictions on data or classify data into categories (Baştanlar\&Özuysal, 2014).

\section{Artificial intelligence in knowledge creation processes}

Knowledge management is the management of corporate knowledge that can improve a range of organizational performance characteristics by enabling an enterprise to be more "intelligent acting" (Wiig, 1993).Knowledge management is "the process of capturing the collective expertise and intelligence in an organization and using them to foster innovation through continued organizational learning" (Meso\&Smith, 2000). Since AI are replicating human intelligence they are used in organisations in interactive systems comprising both humans and AI, it is obvious that, in the general framework of knowledge management in organizations, the AI can be a part of knowledge creation processes. We will investigate how some of the most prominent and notorious applications of AI can be generally considered to have a role in the knowledge creation in organizations, reconsidering the knowledge management goal as to enable people, AI and organizations to collaborate, share, create, use and reuse knowledge. 
Knowledge management shares a goal with the AI community - development and uses computational technology to improve the performance of individuals and communities and the ideas and technology that have been developed by the AI community are required for successful knowledge management (Smith\& Farquhar, 2000). Knowledge management is the process of capturing, processing, distributing, and effectively using knowledge. Bratianu\&Bejinaru (2019, p. 1) mention that knowledge has the main advantage that it has an "invisible contribution to all decision-making and organizational processes", the most important being "knowledge creation, knowledge sharing, and organizational learning", according to Nonaka\&Takechi (in Bratianu\&Bejinaru, 2019, p. 1), Davenport\&Prusak (in Bratianu\&Bejinaru, 2019, p. 1) and Argote (in Bratianu\&Bejinaru, 2019, p. 1).

\section{The dynamic process of knowledge creation (SECI)}

The most highly cited model is the dynamic process of knowledge creation model, also known as the SECI model, of the Japanese authors Ikujiro Nonaka and Hirotaka Takeuchi. This model is a description of the dynamic creation of knowledge in organizations and their practical use, based on the separation between explicit and tacit knowledge. The name of the model is an abbreviation of the four stages that must be passed for the creation of knowledge and their valorization in organisation through applications: S - socialization, E - externalization, C - combination, I internalization (Nonaka\& Takeuchi, 1995).

The main question when analysing a hypothetical SECI cycle comprising humans and AI in a knowledge flow, is where and how can the AI be found in a similar stance as the humans? Can we use AI in all four stages of the dynamic knowledge creation cycle? First of all, there is no real question about the ability of AI to process explicit knowledge, an ability found in any computerised systems. AIs are used in several ways in order to process explicit knowledge and to create new knowledge based on previous knowledge. Hence, the real question is whether the AI can process, in any way, tacit knowledge.

There are examples of relational tacit knowledge being explicated and therefore automated, using for example expert systems and/or neural networks to extract corporate social responsibility values from company documents and match these values to financial outcomes of a company (Sangzoni et al., 2017). Several authors support the idea that the firms' ability to transform big data acquired from social media into knowledge depends on the extent to which they are able to use AI in order to evaluate new information and opportunities, use them to improve their knowledge capabilities, recombine existing information, generate new solutions and add value through knowledge management practices (Cambra-Fierro et al., 2011; De Dreu\& West, 2001; Nguyen et al., 2015; Tiwana\&McLean, 2005). With regard to knowledge elicitation and representation techniques, we can say that AI and knowledge management technologies are often purported to deliver the right information to the right person at the right time (Mohamed \&Zaibon, 2004).

Collective tacit knowledge however, cannot be codified, modelled, or mechanized. AI however is unable to support KM in terms of enabling the circulation of collective tacit knowledge. All AI can do in practice is store articulated rules and apply these to (admittedly increasingly complicated) situations (Sangzoni et al., 2017). To deal with collective forms of tacit knowledge, the subjectivist approach of KM uses social mechanisms such as Communities of Practice (CoP), social networks and action learning (Wenger et al., 2002; Raelin, 2008). 
If we follow, analytically, the SECI cycle, we can see that AI can be used to enhance knowledge management and its knowledge conversion processes. Sharing tacit knowledge (socialisation) can be made electronically without an AI with movies and demonstration of processes stored then made available for any viewer. Bratianu\&Bejinaru $(2019$, p. 7$)$ state that knowledge sharing does not create new knowledge, but changes the knowledge distribution within an organization, due to the fact that it offers access to a larger number of employees to the existing knowledge. "Knowledge sharing is like a diffusion process which increases the overall disorder of organizational knowledge" (Bratianu\&Bejinaru, 2019). Converting tacit knowledge to explicit knowledge (externalization) using AI is possible by expert systems conversion or kinematic analysis of the movements of workers. Knowledge combination (converting explicit knowledge to new knowledge) is the main area where AIs are producing valuable new knowledge by analyzing huge amounts of data. AIs are able to deal with images, text or sounds, are able to codify them in special data that can be used to create results unknown to the developer or the user of the AI. The internalization, made by converting explicit knowledge to implicit knowledge, can be considered to happen during the learning of neural networks in machine learning. The way the neural networks are adapting their answers to the external stimulus, based on the accumulation of data from all previous exposures, can be considered similar to the change in the mental patterns of an individual when exposed to several similar experiences. Machine learning is a subset of AI and focuses on the machine's ability to receive a set of data and learn for itself (Kusunose et al., 2019).

Knowledge as energy metaphor in order to understand the limits of the knowledge as objects metaphor, mostly used in explaining the SECI cycle, and to create an open-minded perspective for the judgments made about the way AI deals with knowledge as a field. In this metaphor knowledge is considered a field; "knowledge manifests in several forms"; "each form of knowledge can be transformed into another form of knowledge"; and "knowledge dynamics means knowledge transformation” (Bratianu, 2018; Bratianu\&Bejinaru, 2020).

\section{Artificial intelligence impact in knowledge management}

The impact of AI in KM is multiple and diverse, yet, without considering this a complete list, we can state that AI has an impact in making available and fastening the processing of big data in real time, simplifying knowledge discovery, making knowledge more easily available, improving the relation with customers, keeping the knowledge base content up-to-date, improving the management of the company.

AI makes available and fastens processing of big data in real time. AI are often responsible to process the enormous amount of data currently circulating in companies locally, nationally or internationally, between individuals or branches, and to rapidly transform them into useful knowledge. AI discovers meaningful and useful patterns in large volumes of data of any type, including text, images, video and other unstructured data. AI simplifies knowledge discovery by using semantic search, natural language processing, and machine learning, to make it easier for employees to find the knowledge they're looking for quickly and easily. AI delivers intuitive search capabilities into the organizational knowledge base. Semantic search and natural language processing in AI lets employees search the knowledge base of the organization using natural language. Machine learning is used to monitor both the terms used in the searches and the user behaviours over time, creating predictions about what employees are looking for. AI makes knowledge more easily available by connecting data from disparate sources. AI helps employees 
when they don't know where to look for the knowledge they need. AI-powered software help employees connect and combine knowledge across multiple systems, giving all employees access to knowledge regardless of where the knowledge is stored.

AI can improve dramatically the relation with customers, by creating and using new knowledge about the customers' behaviours and customers' needs. There are examples of AI used in Chatbots, mapping possible answers learned from previous conversations, in search engine optimization, to improve organic search performances, in content marketing, to personalize content output for each user and generally, to adapt to the user's needs based on learning from his previous behaviours. AI recognizes unique insights about archetypes in the data that allow optimal customer segmentation and treatments. (Akerkar, 2019).

AI helps keeping the knowledge base content up-to-date by using machine learning technology to support employees in finding more up-to-date information. The AI will stop sending people information that are rated by users as unsatisfactory and begin to send something that seems to better satisfy user intent.

AI can improve the management of the company, when used in facilitating or enhancing managerial activity. Self-learning models of AI allows companies to adapt quickly to changes in the patterns of internal or business environment data and underlying business conditions. AIs are really useful in making better and faster decisions by maximizing the value of all your data and moving from predictive analytics to prescriptive analytics (Akerkar, 2019). For example, Posser (2019, p. 1)considers the main tools for business knowledge using AI are: i) Evie for Simplifying meeting scheduling, ii) Aiden AI for Empowering growth; iii) Datasine for Personalising email content; iv) Attest for validating plans and strategy; v) Unito for managing the work management; vi) Freeagent for simplifying finance and accounts; vii) Bizplan for perfecting the business plan.

AI enhances organizational capability by exceeding the human abilities in complex inferential reasoning (by Expert systems), dealing with incomplete data (by distributed information processing in artificial networks), adapting and improving the chances of success in an unpredictable environment (by intelligent agents) and in creating new solutions (by Casebased reasoning). The key advantages of AI over human intelligence are its scalability, longevity and continuous improvement capabilities. Such attributes are anticipated to vividly increase productivity, lower costs and reduce human error (Akerkar, 2019).

AI accelerates innovation by contributing to the creation of new business models and by simple value creation, through the discovery of new patterns in data and fully utilizing knowledge assets (Akerkar, 2019). One particular way of accelerating innovation is by expediting learning, in two ways. First, faster processing of huge available new data creates results transferred to the employees as basis for new insights about the way the activities can be performed. Second, AI can be used in accelerating product design by machine learning used in improving prototyping and in trial and error simulated experiments.

AI improves knowledge management by providing real-time metrics with tool tracking data production, data processing and effectiveness of digitization of complex operations (Joshi \&Natarajan, 2018).

All these kinds of recognized impact of $\mathrm{AI}$ in managing knowledge are arguments for the fact that "artificial intelligence has already brought a huge contribution to the excellence and effectiveness of $\mathrm{KM}$ in term of thinking and problem solving methods, but also through knowledge acquisition, modelling and processing, decision support systems, intelligent tutors, planning, scheduling and optimization systems"(Mercier-Laurent, 2015). 


\section{Conclusion}

Until now, as any computers, AI machines are able to process explicit knowledge, no matter how it is codified. But AI machines are not able to learn collective or individual tacit knowledge, since processing the data about the environment, provided by sensors connected to the AI requires a codification of this reality, similar to the process of expressing knowledge into a explicit form. Still, if imitation is a strategy used by humans to transfer tacit knowledge, replicating the behaviour of a co-worker without understanding it at the beginning, then AI can be considered as able to extract tacit collective or individual tacit knowledge by machine learning mechanisms, when repeatedly being exposed to the practices of organizations or individuals in form of images, words or figures inputs.

AI use in knowledge creation is not meant to replace humans, but is creating opportunities for assisting humans and organizations to perform at a higher level. The core concept of AI focuses in making machines more useful to humans by doing what they do best: very complex and very fast data processing. AI augments knowledge by learning faster the best paths to the right answer, optimizing the process to get the answer, personalizing the answer, or automating the interaction with the environment completely.

By the value created in helping humans learning faster and creating innovation faster, AI technology is expected to introduce a new standard for corporate productivity, competitive advantage and, ultimately, economic growth (Akerkar, 2019).

\section{References}

Akerkar, R. (2019). Artificial Intelligence for Business. Cham: Springer.

Artificial Intelligence - What It Is and Why It Matters. Retrieved from https://www.sas.com/en_us/insights/analytics/what-is-artificial-intelligence.html.

Baştanlar, Y., \& Özuysal, M. (2014). Introduction to machine learning. In miRNomics: MicroRNA Biology and Computational Analysis (pp. 105-128). Totowa, NJ: Humana Press.

Bratianu, C. (2018). Intellectual capital research and practice: 7 myths and one golden rule. Management \& Marketing - Challenges for the Knowledge Society, 13(2), 859-879.

Bratianu, C., \& Bejinaru, R. (2020). Knowledge dynamics: a thermodynamics approach. Kybernetes, 49(1), 6-21.

Bratianu, C., \& Bejinaru, R. (2019). The Theory of Knowledge Fields: A Thermodynamics Approach. Systems, 7(2), 1-12.

Bringsjord, S., Bello, P., \& Ferrucci, D. (2001). Creativity, the Turing Test, and the (Better) Lovelace Test. Minds and Machines, 11, 3-27.

Cambra-Fierro, J., Florin, J., Perez, L., \&Whitelock, J. (2011). Inter-firm market orientation as antecedent of knowledge transfer, innovation and value creation in networks. Management Decision, 49(3), 444-467.

Copeland, B.J. (2019). Artificial intelligence. Enciclopedia Britanica, Retrieved from https://www.britannica.com/technology/artificial-intelligence.

De Dreu, C.K.W., \& West, M.A. (2001). Minority dissent and team innovation: the importance of participation in decision making. Journal of Applied Psychology, 86(6), 1191-1201.

Gams, M., Gu, I. Y. H., Härmä, A., Muñoz, A., \& Tam, V. (2019). Artificial intelligence and ambient intelligence. Journal of Ambient Intelligence and Smart Environments, 11(1), 7186.

https://learnersdictionary.com/definition/learning. 
Joshi, H., \& Natarajan, G. (2018). Digital Transformation through Data and KM. Retrieved from https://www2.deloitte.com/content/dam/Deloitte/in/Documents/technology-mediatelecommunications/in-tmt-knowledge-management-and-big-data-noexp.pdf.

Kusunose, K., Haga, A., Abe, T., \& Sata, M. (2019). Utilization of Artificial Intelligence in Echocardiography, Circulation Journal, 83(8), 1623-1629.

Lemaignan, S., Warnier, M., Sisbot, E. A., Clodic, A., \& Alami, R. (2017). Artificial cognition for social human-robot interaction: An implementation. Artificial Intelligence, 247, 4569.

Mercier-Laurent, E. (2015). Artificial intelligence for successful Kflow. In IFIP International Workshop on Artificial Intelligence for Knowledge Management (pp. 149-165). Cham: Springer.

Meso, P., \& Smith, R. (2000). A resource-based view of organizational knowledge management systems. Journal of knowledge management, 4(3), 224-234.

Mohamed, S. F., \& Zaibon, S. B. (2004). Artificial intelligence support for knowledge management in construction, Retrieved from http://repo.uum.edu.my/13901/.

Moor, J. (2003). The Turing test: the elusive standard of artificial intelligence (Vol. 30) Ed. 2003. Springer Science \& Business Media, Kluwer Academic.

Nguyen, B., Yu, X., Melewar, T.C., \& Chen, J. (2015). Brand innovation and social media: Knowledge acquisition from social media, market orientation, and the moderating role of social media strategic capability. Industrial Marketing Management, 51, 11-25.

Nonaka, I., \& Takeuchi, H. (1995). The knowledge-creating company. How Japanese companies create the dynamics of innovation. Oxford: Oxford University Press.

Pfaffenberger, B. (1993). Dicionario dos usuarios de micro computadores. Editora Campus Rio de Janeiro.

Posser, D. (2019). Seven AI-Powered Tools To Help Start-ups Grow. Retrieved from https://www.forbes.com/sites/davidprosser/2019/07/26/seven-ai-powered-tools-to-helpstart-ups-grow/.

Raelin, J. (2008). Work-Based Learning: Bridging knowledge and action in the workplace. Learning and Teaching in Higher Education, 4-1.

Robbins, S. P. (2003). Organizational behavior. Saddle River, NJ: Prentice Hall.

Russell, S. J., \& Norvig, P. (2016). Artificial intelligence: a modern approach (3rd ed.). Malaysia: Pearson Education Limited.

Sangzoni, L., Guzman, G. \& Busch, P. (2017). Artificial intelligence and knowledge management: questioning the tacit dimension. Prometheus - Critical studies in Innovation, 35(1), 37-56.

Shekhar, S. S. (2019). Artificial Intelligence in Automation. RESEARCH REVIEW International Journal of Multidisciplinary, 4(6), 14-17.

Smith, R.G., \& Farquhar, A. (2000). The Road Ahead for Knowledge Management An AI Perspective. AI Magazine, 21(4), 17-40.

Spence, K. W. (1950). Cognitive versus stimulus-response theories of learning. Psychological Review, 57, 159-172.

Tiwana, A., \& McLean, E.R. (2005). Expertise integration and creativity in information systems development. Journal of Management Information Systems, 22(1), 13-43.

Wenger, E., McDermott, R., \& Snyde, W. M. (2002). Cultivating communities of practice: a guide to managing knowledge. Boston, Massachusetts: Harvard Business School Press.

What Is Artificial Intelligence? How Does AI Work? Retrieved from https://builtin.com/artificialintelligence.

Wiig, K. M. (1993). Knowledge management foundations: thinking about thinking: how people and organizations create, represent, and use knowledge (Vol. 1). Arlington, TX: Schema press.

DOI: 10.2478/picbe-2020-0056, pp. 597-606, ISSN 2558-9652| Proceedings of the $14^{\text {th }}$ International Conference on Business Excellence 2020 